\title{
The potential of GEOTRACES Zn data for constraining biogeochemical model behavior
}

CLAUDIA EISENRING ${ }^{1}$, GREGORY DE SOUZA ${ }^{2}$, SOPHY OLIVER $^{3}$, SAMAR KHATIWALA $^{3}$ AND DEREK VANCE ${ }^{1}$

${ }^{1}$ ETH Zürich

${ }^{2}$ ETH Zurich

${ }^{3}$ University of Oxford

Presenting Author: claudia.eisenring@erdw.ethz.ch

As a consequence of the global ocean circulation and enhanced zinc $(\mathrm{Zn})$ uptake by phytoplankton in the Southern Ocean, the oceanic $\mathrm{Zn}$ distribution correlates with Si rather than with $\mathrm{PO}_{4}$ on a near-global scale [1]. However, the relative importance of additional processes potentially affecting the marine $\mathrm{Zn}$ distribution remains debated $[2,3]$. While dataconstrained models can help quantify the importance of various biogeochemical processes, the sparsity of observational data makes it difficult to constrain model parameters. Additional problems arise from the assumption that analytical observations are error-free, that seasonal variability is insignificant, and that the circulation model is perfect. The resulting high uncertainty is ideally addressed by determining model parameters through mathematical (or objective) optimization methods.

Here, we investigate the potential of the GEOTRACES Intermediate Data Product 2017v2 (IDP2017v2) [4] for constraining four biogeochemical model parameters in a simple Zn-cycling model [1]. We do so via a suite of 30 optimizations to synthetic data, using the Covariance Matrix AdaptationEvolution Strategy (CMA-ES). Our main focus is to assess how optimization results are affected by the spatial and temporal data coverage of IDP2017v2, and by the choice of misfit function. We also assess how the conventional assumption of error-free observational data might affect the optimum solution.

Our results show that optimization with the data coverage for $\mathrm{Zn}$ from GEOTRACES IDP2017v2 leads to very similar biogeochemical properties as when the algorithm is provided with perfect data coverage. However, with increasing complexity of uncertainty in the data, or systematic biases in circulation, the results become strongly dependent on the choice of misfit function. Our work thus suggests that it is possible to reconstruct biogeochemical behavior from the GEOTRACES observational dataset, but also reinforces the importance of the underlying circulation model and prior knowledge of bounds on biogeochemical parameter values.

[1] Vance et al. (2017), Nature Geosci. 10.1038/ngeo23890 [2] Weber et al. (2018), Science 10.1126/science.aap8532 [3] Lemaitre et al. (2020), EPSL 10.1016/j.epsl.2020.116216 [4] Schlitzer et al. (2018) Chem. Geol. 10.1016/j.chemgeo.2018.05.040. 